\title{
UPCommons
}

Portal del coneixement obert de la UPC

http://upcommons.upc.edu/e-prints

This is a post-peer-review, pre-copy edit version of an article published in Computing. The final authenticated version is available online at: https://doi.org/10.1007/s00607-018-0680-z.

\section{Published paper:}

Poniszewska-Maranda, A., Kaczmarek, D., Kryvinska, N., Xhafa, F. Studying usability of $\mathrm{Al}$ in the IOT systems/paradigm through embedding NN techniques into mobile smart service system. "Computing", 2018. doi:10.1007/s00607-018-0680-z

URL d'aquest document a UPCommons E-prints:

https://upcommons.upc. edu/handle/2117/127081 


\title{
Towards Building Mobile Smart-IoT Service System
}

\author{
Aneta Poniszewska-Maranda • Daniel \\ Kaczmarek • Natalia Kryvinska • Fatos \\ Xhafa
}

Received: date / Accepted: date

\begin{abstract}
The Internet of Things (IoT) has emerged as a disruptive technology for the current and future of computing and communication. IoT is characterized by a variety of heterogeneous technologies and devices able to be connected to the Internet. Current and future research and development efforts aim at adding artificial intelligence to IoT systems, enabling devices to become smart and thus make autonomous decisions individually or collectively. Additionally, such smart devices have the ability to interact not only with other smart devices but also with humans. Thus, the aim of this paper is to investigate the usability of the artificial intelligence in the IoT paradigm. To achieve the approach, a system called smart-IoT is built based on artificial neural networks, namely, neural networks have been learned by back-propagation algorithm. The system is tested using mobile devices under Android as smart objects. Experiments with neural networks were carried on certain services (such as auto set alarms for a specific event, or estimating the time to return home). These experiments showed the feasibility of embedding neural networks
\end{abstract}

\author{
Aneta Poniszewska-Maranda \\ Lodz University of Technology \\ Lodz, Poland \\ E-mail: aneta.poniszewska-maranda@p.lodz.pl \\ Daniel Kaczmarek \\ Lodz University of Technology \\ Lodz, Poland \\ E-mail: dkdaniel@vp.pl \\ Natalia Kryvinska \\ Comenius University in Bratislava \\ Bratislava, Slovakia \\ E-mail: Natalia.Kryvinska@fm.uniba.sk \\ Fatos Xhafa \\ Universitat Politècnica de Catalunya \\ Barcelona, Spain \\ E-mail: fatos@cs.upc.edu \\ * Corresponding author
}


techniques into the IoT system. The approach allows also for easy adding of new services, which in turn means that smart IoT is a modular and full-fledged system.

Keywords Artificial Intelligence (AI) - Neural Networks · Internet of Things $(\mathrm{IoT}) \cdot$ Mobile Device $\cdot$ Mobile Service System · System Design

\section{Introduction}

The Internet is nowadays a powerful tool used in all IT systems. The salient feature of the Internet is its ubiquity, namely, the network is available at home, at the university and on portable, small devices (phones, watches), sensors, etc. The ubiquity and the ever increasing improvement of small devices both in terms of connectivity and computaional resources have lead to the Internet of Things (IoT). It is estimated that up to 15 billion of different devices are currently connected to the Internet, where this can be less than $1 \%$ of what might actually be connected to the network [8, 9, 13, 19, 28].

The purpose of this paper is to investigate how Artificial Intelligence (AI) techniques and methods can be used in the Internet of Things (IoT) paradigm to endow IoT systems with various degrees of intelligence. It should be noted here that given the limitations of IoT devices, it is not clear which AI techniques are suitable for what kind of IoT systems. While we investigate the above issue from a general perspective, on the one hand, to make the study concrete, here we consider mobile devices, typically mobile phones under Android, as an intelligent object for the IoT system; on the other hand, we consider Neural Networks with back-propagation as a main AI method implemented in this system. In addition, a review of current state of the art related to the problem is presented, by critically analysing their advantages and disadvantages. The paper covers also some software engineering issues for building IT systems, using mobile devices and AI methods (issues of system security in terms of data management are not considered here).

The reminder of the paper is organised as follows. We start with an analysis of mobile devices as intelligent objects in the Section 2 A detailed analysis of the functionalities and elements of the created system is given in the Section 3 . It covers the basic definitions as well as the architecture, communication of the objects. We continue in Section 4 with an examination of selected AI techniques by exploring general issues related to AI along with a more detailed presentation of selected methods used to implement the IoT prototype. Next, in Section 5. a technical sketch of the IT prototype is presented, where the specific functionality of the system is detailed. An analysis of the operations of the system along with its evaluation is given in Section 6. Finally, in Section 7. a summary of the research findings, challenges of the problem, and further research directions as well as implementation areas are discussed. 


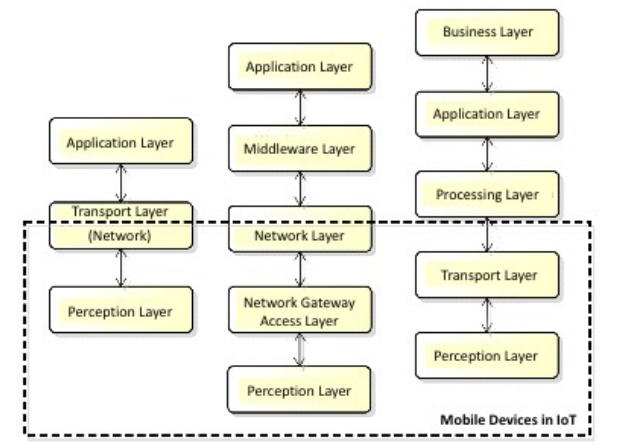

Fig. 1 The location of mobile devices in the context of IoT architectures.

\section{Mobile Devices as Intelligent Objects}

The number of devices used in systems based on the IoT concept is exponentially growing. It is now becoming commonplace that a person is surrounded by many devices that can assist him and make some decisions either automatically or through an interaction process. To be able to achieve the best out of the IoT devices capabilities, they should be smart, therefore the field of Artificial Intelligence (AI) is crucial to achieve smart IoT. However, the nature and limitations of IoT devices require the right choice of AI methods in order to increase the intelligence and the accuracy of the decision, while ensuring intuitive and easy-to-use human interfaces and communications [1, 10, 17, 30, 31 .

To make the discussion concrete, we consider here the use of artificial intelligence in IoT systems based on the mobile devices as smart objects. It should be noted that there is a great variety of smart devices, among which mobile devices are among most important ones. According to the IoT concept, mobile devices, e.g. mobile phones, can be treated as intelligent objects. Indeed, they have built-in sensors, a place for local data storage, and communication technology elements, among others. Fig. 1 shows the location of mobile devices in the context of the IoT architecture. It can be said that regardless of the architecture chosen, mobile devices fully support elements associated with the lowest layers of perception. Furthermore, without the need to add supplementary physical devices to the architecture, and only with internal software, components such as pre-processing and local storage can communicate with other intelligent devices in the system, and transmit data to the gateway (to deliver to relevant applications, services or servers). Thanks to this view of mobile devices, they can be easily integrated into information systems based on IoT [2,4, 16, 20, 22, 27, 29].

In fact, mobile devices are hybrid devices in the IoT context sonce they can also play the role of a computer. In addition to using them to only collect sensor data they have a variety of sensors (up to a dozen) different applications that may be able to manage other IoT objects can be developed. This allows them 
to be aggregators of network traffic from multiple sensors. These devices have significant processing capabilities and can also act as gateways to platforms offering various services $33,34,35$.

In the following sections we focus on endowing IoT systems with artificial intelligence, and propose a system to assess the suitability of selected artificial intelligence methods for use in the IoT paradigm.

\section{Operation of IoT systems with artificial intelligence}

Systems built on IoT concept use not only simple sensors transmitting information to systems that primarily operate on the basis of statistics and simple mathematical calculations. Such systems are increasingly being developed for making decisions on different aspects and contexts. It is easy to imagine a system that turns on heating only on the basis of ambient temperature. However, it could regulate the temperature also in terms of the number of people in the room, the habits of a user, specific rooms or daytime. Therefore, endowing intelligence to these systems is an important issue, yet rather complex. In the forementioned example of ambient temperature and user habits makes us aware that the IoT system should somehow learn about these users habits and adjust to them. Such actions are not to be achieved by simple statistics or simple equations. Here we need more refined and complex tools such ones based on artificial intelligence 6, 7, 11, 16, 21, 26

The idea of using artificial intelligence in the Internet of Things is a further step - namely, a question of the independence of machines in the context of their supervision. The use of AI methods can have advantages on decision making either autonomously by IoT system or in communication with humans 23,24 .

\subsection{Architecture of Smart IoT system}

Endowing the IoT system with AI arises the issue of its location in the IoT architecture. There are several aspects to be taken into account, such as the performance and the amount of data space that is certain system knowledge, so AI techniques will be allocated to adequate levels. Fig. 2 shows the general idea of embedding AI in the context of general IoT architectures [14, 18, ?].

AIs most natural places are the servers of all types because of their computing power. This location positively affects one more aspect - reusability. The place of action of various AI tools can be compared to the human brain. Due to the fact that all knowledge and related inference and learning are placed in the servers, it is possible to use them in a broader perspective. For example, a subsystem using AI tools can react on a number of devices and sensors, and thus build a structure that will provide intelligence for all interrelated components. Such an approach can respond to changes in the global environment, so that it can better adapt to changes. Of course, a location of artificial intelligence in the servers does not exclude the possibility of placing it directly 


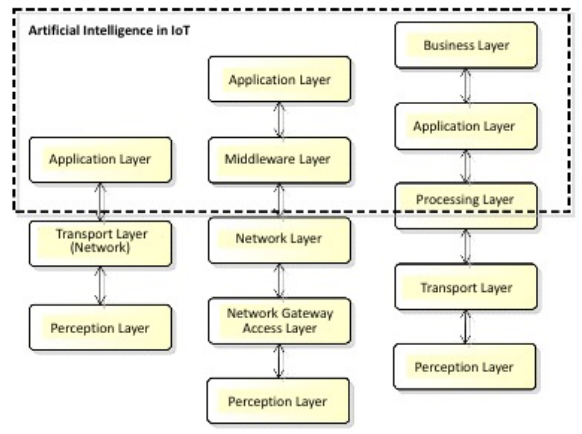

Fig. 2 Placement of artificial intelligence in IoT architectures.

in devices, but a general set of knowledge available throughout the system greatly enhances its intelligence capabilities. Some artificial intelligence components can be distributed directly to the devices, depending on the specific application and problem to be solved [1,2,3].

Fig. 3 shows a general flow of information in IoT systems with AI. Blue arrows display data sent from the real world to different (parts of) systems. Red arrows show data processed by systems that already have the right context, and to which IoT systems and devices can respond appropriately (make the right decisions). Black arrows represent internal communication, understood as an additional communication channel between intelligent objects themselves. This channel is a communication bus that can send data over the Internet, but does not need to (other technologies such as bluetooth are used). These are three steps to share communication with, they represent key elements of the entire IoT system operation using artificial intelligence [1,2,13].

\subsection{Preliminary communication}

The first stage can be called pre-communication (blue arrows in Fig. 31). At the beginning, according to the IoT concept, data from the environment (real world) is collected by IoT devices. These can be external sensors as well as those built into the device. They are then pre-processed to be legible for the rest of the system. Depending on the specific case, data is transmitted over the Internet to major IoT system or between other devices to gather all relevant information from the real world (internal communication elements) [33, 35. In the context of mobile devices, it is also important to temporarily store item information when there is no access to the Web. When all data, in the context of a specific cycle, are already in the IoT system, they are properly prepared for the external AI system. This process may include an appropriate selection of specific information to be targeted for a particular AI tool (for example, ambient temperature and number of people as input parameters to the neural network in the temperature control system) [30,32. 


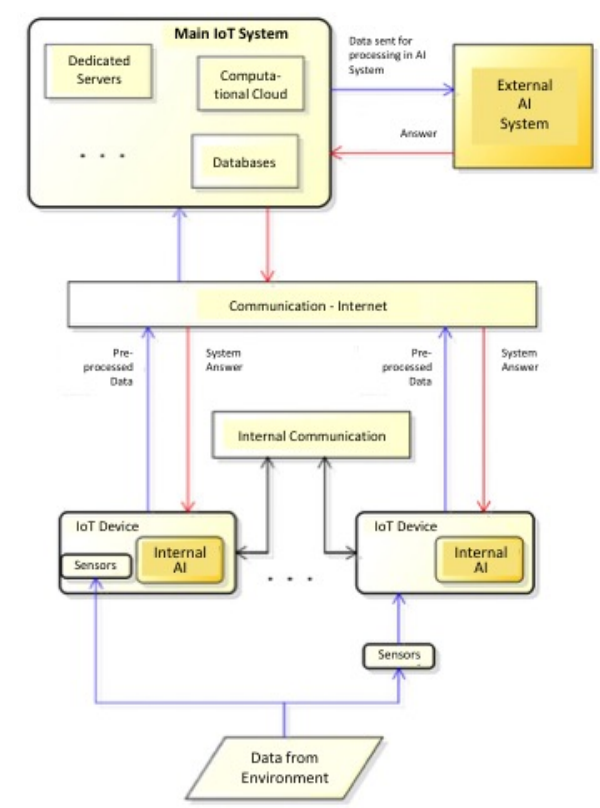

Fig. 3 General flow of information in IoT system with AI.

\section{Contextual communication}

The next stage of communication is a contextual communication (red arrows). Its main purpose is to provide specific system responses to IoTs and subsystems that are supposed to take concrete action in the context of the relevant decision. When the AI processing ends and gives a certain answer, it should be appropriately interpreted. This task should perform the main IoT system. It is often the case that the AI response is a numerical value, which, without relevant context, makes no sense. Therefore, an important step is to link them with the knowledge base so that the overall response of the system to the specific problem can be deduced, and thus responded accordingly, i.e. to make a smart decision. Data found in major IoT system should be interpreted properly and could usually be sent to some of IoT devices so that they can respond appropriately. It is also possible to imagine a situation where processed data must be sent to other IoT devices than those from which the original data originated. An example may be a monitoring system. Devices that have cameras send real-time data to major IoT systems that, once a specific threat has been detected (use of AIs), can send data to other IoT devices that may respond to such threats in some way [2, 12 .

\section{Internal communication}

Internal communication (black arrows) is an additional communication. In the proposed model, it can be placed both in the pre-communicative and the con- 


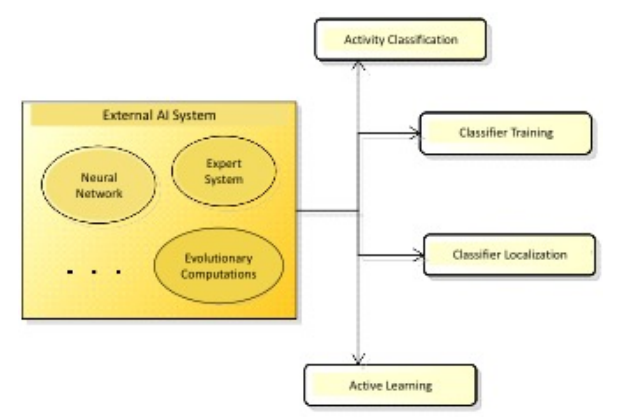

Fig. 4 Main elements of AI systems in the IoT concept.

textual stage. In the first case, it can be used to gather enough data from multiple devices and send them simultaneously to higher system layers. In the latter case, having already made a specific response from the system, it can be used to work together with multiple devices simultaneously. Such an approach, combined with additional AI elements within the devices themselves, can introduce more aspects of autonomy into smart objects [2,12,

\section{Artificial Intelligence-based IoT}

The above stages are related to the general description of IoT systems using artificial intelligence. The following are directly related to AI systems. Fig. 4 shows schematically the main elements of the external AI systems, as key elements in the IoT concept. External AI systems primarily include a variety of AI methods (e.g. artificial neural networks or expert systems) that are responsible for various types of intelligent decision-making operations based on the knowledge acquired and for supporting other simpler systems those are part of the whole IoT system. In addition to the specific AI implementations, the four main elements of artificial intelligence, which have a significant impact on the performance of IoT systems, are directly related to the behaviour of the systems themselves (mainly for the sake of learning):

- Activity Classification is an action in which sensor data is transferred to a learning algorithm in the form of isolated features that are calculated after initial data processing. Classification functions are used for this purpose during pre-processing. The key element here is an appropriate labeling of the data set (for example, by semantic labeling) that comes from the real world. This allows the data to have extracted elements (traits) that are essential in the context of machine learning and later system operation. This action selects the most important features and is one of the main AI components in the IoT system [2].

- Classifier Training is a process of building general models of reasoning. To build the right model it needs the right knowledge that is acquired from an environment. Building classifiers for IoT is crucial for inference and 
decision-making from various types of intelligent objects. Another important aspect is also training. In the case of system operation and retrieval of new data, the system may be able to self-classify the classifiers and investigate their accuracy without user intervention (for example, systems based on a time-element detection) 2 .

- Classifier Location refers to specific location of the learned classifiers. Their placement must be thought out mainly because of the high computational cost. Classifying data from a large number of devices, apart from high computational cost, can endanger the scalability of the entire system. Therefore, it is important to separate the main artificial intelligence methods into external systems that have high computing power and leave inside only those supporting IoT devices (if needed). Equally important at this point is the initial data processing so as not to send them directly from the sensors (raw data). The purpose of preprocessing the data is to adapt them for communication protocols, which directly relate to the reduction of the volumes transfer, thus reducing the bandwidth required (e.g. data fusion methods can be employed).

- Active Learning is often referred to as learning by inquiry and is an additional, albeit equally important, element. Just like in the case of teaching classifiers, here we can talk about training. Active learning involves asking the user whether the system has made the right decision, and if not, where he has gone wrong. With such knowledge, AI systems can rebuild their inference model to avoid similar errors in the future. Examples may be various types of recommendation systems that may take into account user feedback on previous recommendations 2,25.

The above elements show that an appropriate integration of AI methods into IoT architecture arises many challenges and problems to be addressed.

\subsection{Integration of AI Methods into IoT Architecture}

To investigate an actual usefulness of AI methods in the IoT concept, an IT system has been developed, the technical aspects of which are discussed in this subsection. Functional and non-functional requirements of this system are discussed in Sections 4.1 and 4.2 The proposed system (hereafter, smart-IoT) aims to study the time of return home and set alarms at an appropriate time for a given event. The proposed system consists of three main components:

- Mobile devices - smart objects

- Central server - the main IoT management system

- Micro-sites - contain elements related to AI methods

Fig. 5 (left) shows a general scheme of the prototyped system, which is based on 4-layer architecture:

- Perception layer - its main task is to collect data from the environment using built-in sensors in cell phones (GPS, accelerometer, and gyroscope) and their initial processing. 


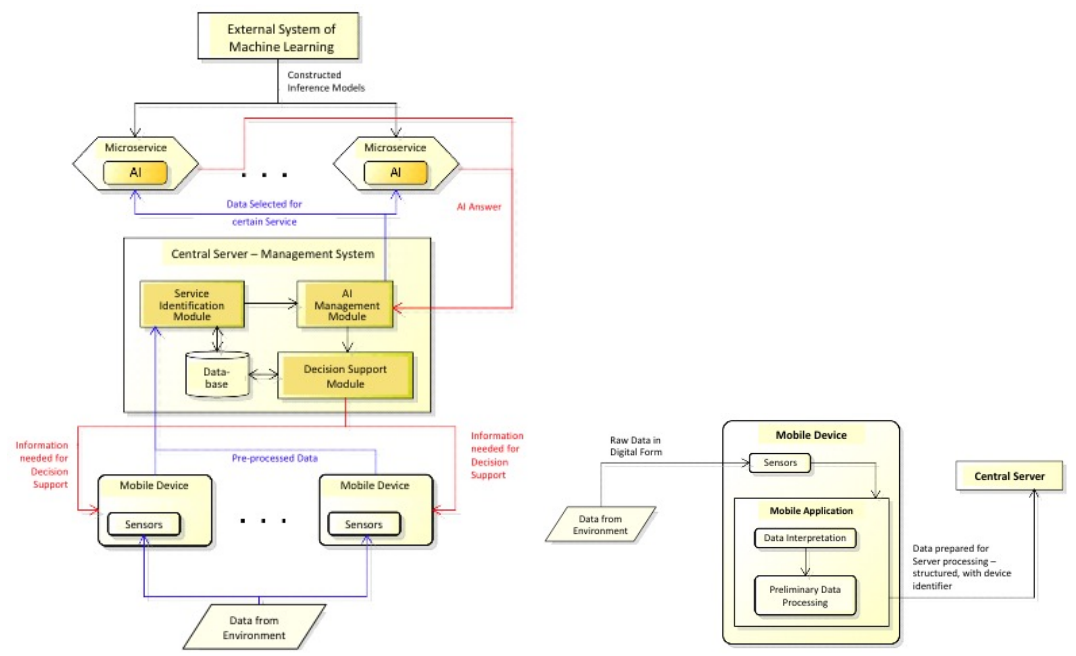

Fig. 5 The scheme of smart-IoT system (left). Schematic operation of smart objects in smart-IoT system (right).

- Network layer - responsible for transferring data between all system components. IEEE 802 protocols (in particular LAN and Wi-Fi networks) are used.

- Processing layer - is an essential part of the whole system. This layer is responsible for processing the information in the central server with the proper use of micro-services, i.e. AI methods. Its primary purpose is to process input data and generate a specific response for them in the context of the service.

- Application layer - is responsible for presenting the data to the end user. Its main role is to keep the data up-to-date (updated accordingly from the decision-making module).

The following subsections present its technical aspects in the context of specific elements.

\subsection{Intelligent objects in the system}

Intelligent objects in the proposed system are mobile devices (specifically mobile phones). Their main task is to collect relevant data from the real world using built-in sensors. The next step concerns the initial processing of the data. All these tasks are performed using a dedicated mobile application. According to the IoT concept, all devices must be uniquely identifiable throughout the system. To that end numeric identifiers are used. Each mobile device transmits a unique identifier, which is then recorded on the central management server. This approach also allows to limit access to unauthorized devices, as the system will only support the information that is stored in a user database. Fig. 5 (right) shows the diagram of smart objects. 
A dedicated mobile application, in addition to storing a given device identifier, also has elements that identify the service they are performing. This is to ensure versatility. After receiving data from the sensors, the application must recognize their destination (choose which services are intended for data consumption). The next step is then to prepare them in a readable form for a central server. For this purpose, it is proposed to use the HTTP protocol. For the server to be able to properly interpret the data, send the following items:

- The identifier of the device from which the information originates,

- Service ID, so that the central server can call the appropriate method (one sensor can use multiple services)

- Described environmental data (for example GPS data as latitude and longitude).

Such elements form the basis of the entire process that occurs at the level of intelligent objects.

\subsection{Main IoT components}

The main components of IoT system are implemented at a central server acting as a manager. It consists of four essential elements:

- Service Identification Module

- AI Management Module

- Decision-Making Module

- Database

The Service Identification Module is responsible for properly interpreting the incoming data from the devices. Its key task is to group data by type of service and device. For this purpose, it uses a database connection, where all configurations of the registered devices are stored (information about the services the device uses and corresponding AI tool used). Another element is the AI management module. It receives grouped data from the service identification module so that it can send the appropriate parameters to the appropriate micro-services, which are used for AI calculations. Upon receipt of the response, the data is sent to the decision-making module that performs the operations of the service. This can be a simple display of information on a central system or sending data to an IoT device that is supposed to take a user decision (in the context of the smart-IoT system the alarm setting time). The operation flow of the central server is schematically shown in Fig. 6 (left).

\subsection{Components of artificial intelligence}

Intelligent components are responsible for intelligent calculations in the context of AI methods. For the smart-IoT system, it is proposed to use microservices as a service for specific methods. The micro-service assumes the sharing of one specific service (for example, the implementation of a particular 


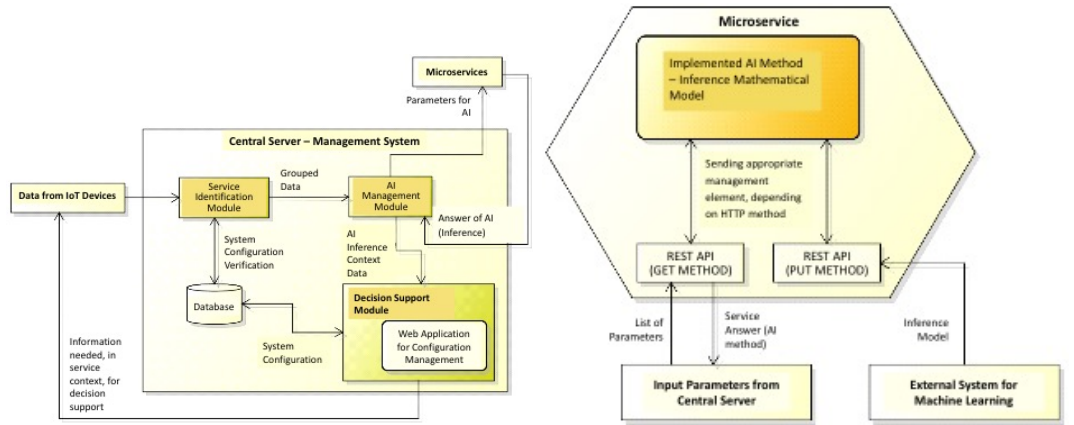

Fig. 6 Description of central management server operation in smart-IoT system (left). Micro-service diagram of smart-IoT system (right).

artificial neural network model). With this approach, the central server does not need to know exactly what element of artificial intelligence is used to solve a problem but to know its existence through an ID. This approach can provide a quick adjustment of AI methods in the context of the device. The REST (Representational State Transfer) interface has been proposed for communication with micro-services.

In the context of REST an important concept is the resource. In the context of the smart-IoT system, resources can represent specific micro-services, so artificial intelligence is implemented. In addition, each resource can be invoked by one of the four interchangeable methods, so that other effects can be derived for the same URI (for example, calling a micro-site in the context of neural network training or in the context of obtaining a particular result in the inference process). In addition, in the context of AI components, an external machine learning program was proposed. Its goal is to train appropriate AI tool, and build on it a mathematical model of reasoning. Such a model is placed in micro-sites where it can be used to make decisions for a particular device. Fig. 6 (right) shows a diagram of the operation of micro-services in smart-IoT system. The next section focuses on the practical implementation of this system together with the evaluation of the use of selected AI methods in IoT systems.

\section{Smart-IoT Service System built using AI approach}

We start here by a description of the functional and non-functional requirements and the technologies used for the smart-IoT system.

\subsection{Functional requirements to the Smart-IoT system}

The use case diagram in Fig. 7 (left) illustrates the general functional requirements of the smart-IoT system. 

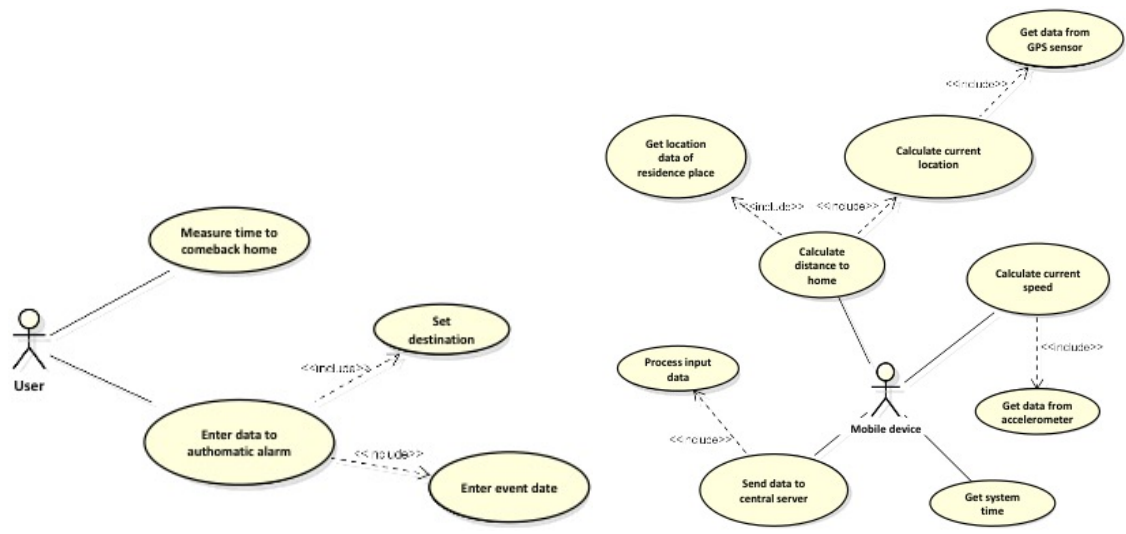

Fig. 7 General use case diagram of smart-IoT system (left). Mobile Device Use-case Diagram for a time-back-home Service (right).

Building a system based on the proposed architecture (see Section 3) has led to the creation of two main functionalities. The first is to measure the time to return home. This functionality is responsible for displaying the home-return time accurately to the minute in real-time, after having entered the location data of the place of residence. The second feature is the automatic setting of an alarm clock on the phone for a specific event. The alarm starts at a predetermined time and the proposed departure time is given so that the user can manage the event (for example, a job or meeting with a client). To determine the alarm and exit times, the system needs input data: the location of the place where the event occurs and the start time of the event.

The main functions (services) of the smart-IoT described above are used to calculate relevant information from selected AI methods (artificial neural networks). Consequently, an additional console application for learning neuronal networks has been developed with the method of error back propagation. A detailed description of the services is presented next.

\section{Use case: The time to return home}

The home-return service uses two sensors in the phone: the GPS module and the accelerometer. The first one collects location data in the form of latitude and longitude, while the second one is used to determine the speed of movement. This service needs input data as the length and latitude of a users residence. Fig. 7 (right) shows the use case diagram for the smart object in the context of the service described. According to the description of the system architecture, such objects must additionally prepare data for the central server.

The data transmitted from the mobile device to the central server includes the following information: length and latitude of place of residence, the latitude and longitude of the current location, current speed of movement and system time. 


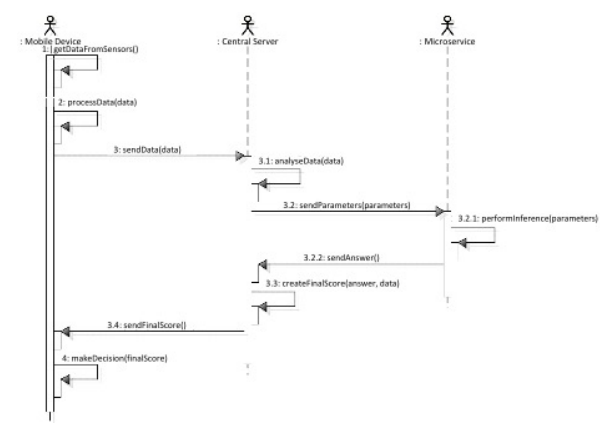

Fig. 8 Sequence Diagram for Home Return Time Service.

The device sends this information to the server intermittently (as specified a number of seconds). With this information, the central server is able to count the distance of residence from the current position, the direction of travel and the offset distance from the last measurement. The archival data from the previous readings is used for the last two items.

This allows the following input to the neural network to be prepared:

- distance of current position from the place of residence - numerical value in meters,

- current time - a numerical value specifying the number of minutes per day, counted from midnight,

- speed of movement - in $\mathrm{km} / \mathrm{h}$ unit,

- shift from the last measurement - measured in meters,

- direction of movement - north, south, east, west,

- direction to the place of residence - as above.

Based on the presented 6 input parameters, the artificial neural network corresponds to a specific numerical value that represents the estimated return time of the user to the home. This value, like the parameter, the current time, is given as the minute number counted from midnight. The server interprets the result accordingly and sends it to the mobile device where a specific response is displayed. In addition, this response is also displayed on the server itself. The process of this service is illustrated in the sequence diagram in Fig. 8.

\section{Automatic alarm setting}

The auto-event alert service is designed to notify you when you should leave your home to reach a specific destination at your desired time. Data about the destination and the time of the planned arrival are needed, based on which, the mobile device will alert the user at the soonest time of the departure.

An additional element here is the so-called make sure you leave the house. A mobile device using the fact of connecting to a home Wi-Fi network (if any) and additionally location data resides periodically, using an alarm, to leave until you leave home. When the device has moved away from home, it 


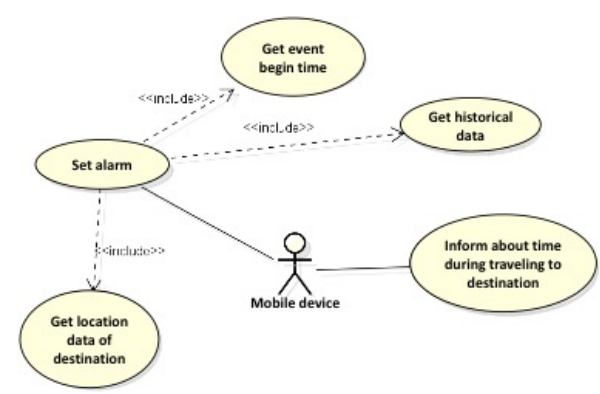

Fig. 9 Mobile Device Use Case Diagram for Automatic Alarm Setting Service.

is assumed that the user has left and goes to the destination. In this case, the phone informs about the so-called time indicator of arrival (messages: ahead of time, in standard, late). Fig. 9 shows a use case diagram for an intelligent object in this service context.

\section{The artificial neural network}

To set the alarm time, the service is based on historical data that is used to train an artificial neural network. The neural network thus constructed has the following input parameters:

- distance of the target position from the place of residence - numerical value in meters,

- time of arrival - the numerical value for the number of minutes per day, counted from the north,

- average time of arrival at similar distance in minutes - data from previous journeys that represent the correct value,

- distance difference in meters - the difference between the first parameter, ie the distance between the target position and the distance found in the archives.

Based on the above input parameters, the artificial neural network calculates a specific result and presents it as a time (minutes from midnight), representing the departure time. Once the server responds to the response, the appropriate time is sent to the mobile device that will start alerting the user at a certain time that the time of departure is approaching. Fig. 10 shows the operation of this service illustrated in the sequence diagram. It is worth mentioning that the elements related to the transmission of the arrival time index use the sequence of action described in Fig. 8.

\subsection{Non-functional requirements of the Smart-IoT system}

For proper operation of the smart-IoT system it should meet the following standard requirements: 


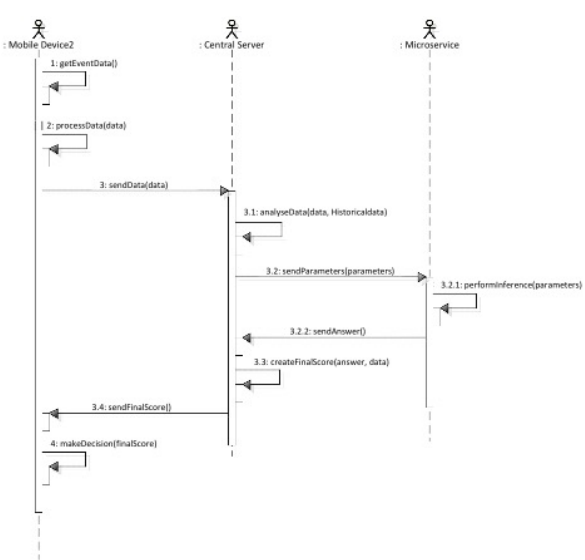

Fig. 10 Sequence Diagram for the Automatic Alarm Setting Service.

- Minimum Android 4.4 phone with GPS, accelerometer, and optional magnetometer - serves as an IoT device (we used a mobile device with 2 GB of RAM and a 4-core $2.2 \mathrm{GHz}$ processor).

- A central server and micro-services installed.

- A wireless Wi-Fi router in a location that was previously described as a home.

\section{Suitability of AI Methods for the IoT Concept}

\subsection{Neural Network Model for Usage in the IoT Applications}

In the context of technical applications, artificial neural network is a collection of interconnected simple processing units whose action is inspired by biological neurons. On the other side, it can be seen as a set of highly parallel and distributed CPUs, consisting of simple computational elements that have a natural tendency to memorize the information provided. All knowledge and capabilities of such network are stored in the form of architecture, and in its weight of connections between units. Weights are determined by the learning process. The knowledge is gained from an environment in the course of learning process.

Schematic representation of the artificial neural network is shown in Fig. 11 In this case, each neuron is connected to each neuron from the next layer. This means that the output of a certain neuron goes to the inputs of the entire next layer. Such a model of neural network is called multilayer perceptron. It is the most commonly used model for solving a variety of problems. We distinguish three types of layers:

- Duplicating layer distributes signal to the first layer hidden in unchanged form, not a processing layer. 


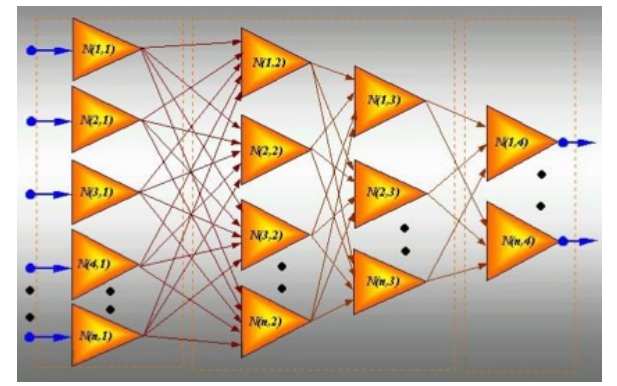

Fig. 11 Model of Artificial Neural Network.

- Hidden layers (or one) they are processing layers. Each neuron of this layer accepts all the neurons output from the previous layer, calculates the sum of the sum and gives the value of the activation function for that sum.

- Output layer the processing layer, but its output is the final response of the network for the given input pattern.

To train such a network the backpropagation error algorithm is most often used. The number of neurons in hidden layers must be well matched. Too few of them cause the inability of the network to accumulate sufficient knowledge about the problem to be solved. Too many neurons in turn cause too much memory training data, which causes problems with generalization of input patterns.

In the artificial neural networks, two phases can be distinguished, the first of which is learning phase, while the other one is a response phase to the specified external stimulus. This network is first trained, and after this process is able to react appropriately to the input element. It is worth mentioning that there are two types of learning: supervised learning and unsupervised learning. The first of them implies some supervision over the stage of learning. It consists in creating a learning set of data pairs input object (e.g. vector) and desired output (e.g. specific numeric value). This type of learning is often used for classification problems. It consists of assigning specific input values to a certain predefined class. With this approach, a neural network builds its internal structure so that it responds appropriately to the input. With good input selection, the network is also able to respond well to a stimuli that it did not know before. An example of a neural network that is taught in a supervised manner is multilayer perceptron (see Fig. 11).

The second type of machine learning, used in artificial neural networks, involves the absence of a teacher (human supervision). This means that a network does not have direct information about the desired response and models the response function on the stimulus itself. Generated responses must be appropriately interpreted in the context of a particular problem. This type of learning is used in the context of grouping. This means that having a certain set of data, the neural network is trying to find a relationship between them. Depending on which features of the objects are taken into account and what is 
the accuracy of grouping, the neural network will recognize a certain number of groups of similar objects, for example - grouping colors in terms of their brightness. An example of a network using unattended learning is the so-called Hopfield network [15].

Backpropagation error algorithm is the most commonly used neural network learning method and is based on supervised teaching of unidirectional multi-layer neural networks as it is one of the most effective methods. Before the operation of the algorithm, all neural network weights are randomized.

In the next section, we exemplify a neural network usage in the IoT system and perform extended experiments. A detailed analysis of results is also given.

\section{Experimental Study and Analysis}

In order to judge on the suitability of selected artificial intelligence methods in the smart-IoT system, namely, artificial neural networks with back propagation, an empirical study through various tests is conducted. For the tests a route of $4 \mathrm{~km}$ was selected. To obtain training data for a neural network, measurements were made at different times of the day. There have been 10 routes (under the same configuration, some of them on foot and some by public transportation). From each run of one route 100 measurements were taken from different parts of it. As a result, the training data contained 1000 items.

These consisted of the following (also previously described) six input parameters: distance of your current position from your place of residence, current time, speed of movement, shift from the last measurement, direction of movement, direction to the place of residence.

For the first measurement, it was assumed that the offset parameter from the last measurement was 0 . For each element containing 6 input parameters, the correct result was known, i.e. the return time to the home. Having all of the above elements, two neural networks, multi-layer perceptrons, reverse-error propagation algorithms were studied. In both cases, a sigmoidal activation function was selected.

The description of the network is as follows:

- A neural network with one hidden layer, called SN1 - a network containing 6 input neurons, 3 hidden neurons and 1 output neuron.

- Neural network with two hidden layers, called SN2 - a network containing 6 input neurons, 3 neurons in the first hidden layer, 2 neurons in the second hidden layer and 1 output neuron.

In addition, an SN3 neural network, which contains 4 input neurons, 3 neurons in the hidden layer and 1 neuron output, was taught for the automatic setting of the alarm. The above network contains the following input parameters (the same set of training data that was tailored to this problem was used): distance of the target position from the place of residence, time to reach the destination, average time to reach a similar distance in minutes, distance difference in meters. 
Table 1 Test results in SN1 and SN2 neural networks for home-return testing service.

\begin{tabular}{|l|l|l|l|l|l|}
\hline & $\begin{array}{l}\text { current } \\
\text { time }\end{array}$ & $\begin{array}{l}\text { distance } \\
\text { to home }(\mathrm{km})\end{array}$ & $\begin{array}{l}\text { actual } \\
\text { time }(\mathrm{min})\end{array}$ & $\begin{array}{l}\text { time given } \\
\text { by SN1 network }\end{array}$ & $\begin{array}{l}\text { time given } \\
\text { by SN2 network }\end{array}$ \\
\hline 1 & $9: 00$ & 4 & 37 & 42 & 40 \\
\hline 2 & $9: 00$ & 3 & 25 & 23 & 26 \\
\hline 3 & $9: 00$ & 2 & 12 & 9 & 12 \\
\hline 4 & $9: 00$ & 3 & 23 & 21 & 21 \\
\hline 5 & $9: 00$ & 2 & 11 & 10 & 9 \\
\hline 6 & $9: 00$ & 1 & 7 & 5 & 6 \\
\hline 7 & $17: 00$ & 4 & 48 & 51 & 45 \\
\hline 8 & $17: 00$ & 3 & 32 & 30 & 30 \\
\hline 9 & $17: 00$ & 2 & 20 & 14 & 17 \\
\hline 10 & $17: 00$ & 3 & 25 & 29 & 29 \\
\hline 11 & $17: 00$ & 2 & 14 & 12 & 11 \\
\hline 12 & $17: 00$ & 1 & 7 & 8 & 7 \\
\hline
\end{tabular}

Table 2 Test results in SN1 and SN2 neural networks for home-return testing service.

\begin{tabular}{|l|l|l|l|l|}
\hline & $\begin{array}{l}\text { meeting } \\
\text { hour }\end{array}$ & $\begin{array}{l}\text { distance to } \\
\text { destination }(\mathrm{km})\end{array}$ & $\begin{array}{l}\text { exit time given } \\
\text { by the network }\end{array}$ & $\begin{array}{l}\text { arrival } \\
\text { time }\end{array}$ \\
\hline 1 & $08: 00$ & 4 & $7: 10$ & $7: 49$ \\
\hline 2 & $08: 00$ & 4 & $7: 14$ & $7: 57$ \\
\hline 3 & $08: 00$ & 4 & $7: 09$ & $7: 52$ \\
\hline 4 & $12: 00$ & 4 & $11: 15$ & $11: 45$ \\
\hline 5 & $12: 00$ & 4 & $11: 20$ & $11: 46$ \\
\hline 6 & $16: 00$ & 4 & $15: 01$ & $15: 55$ \\
\hline 7 & $16: 00$ & 4 & $15: 09$ & $16: 10$ \\
\hline 8 & $16: 00$ & 4 & $15: 03$ & $16: 16$ \\
\hline
\end{tabular}

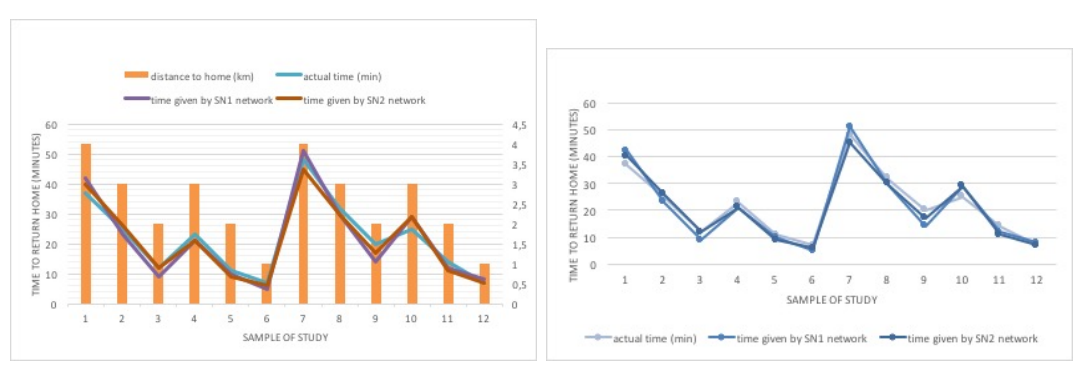

Fig. 12 Comparison of neural networks with actual data in IoT solutions for number of samples (left). Comparison of neural networks with actual data in IoT solutions (right)

Thus, using the neural network learning program, the SN1, SN2 and SN3 networks described above were used to carry out test routes. The first two were used in the context of the home-return time service, while the SN3 network was automatically set to alarms. The results of these measurements are given in Tables 1 and 2, and Figures 12, 13 and 14

Table 1 and Figures 12 13 contain the results of the experiments conducted in the context of the time-to-return service. As can be seen, both neural networks achieved similar results and were similar to those of the actual ones. Also, in both cases, there is a tendency between morning time and afternoon 


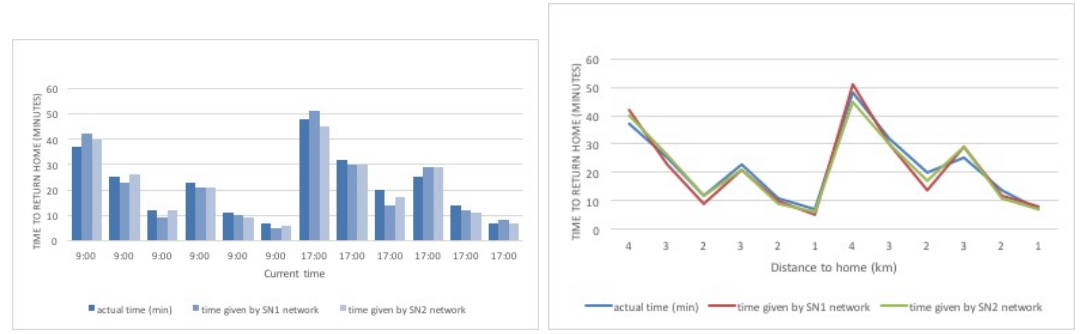

Fig. 13 Comparison of neural networks with actual data in IoT solutions for current time (left). Comparison of neural networks with actual data in IoT solutions for given distance to home (right).

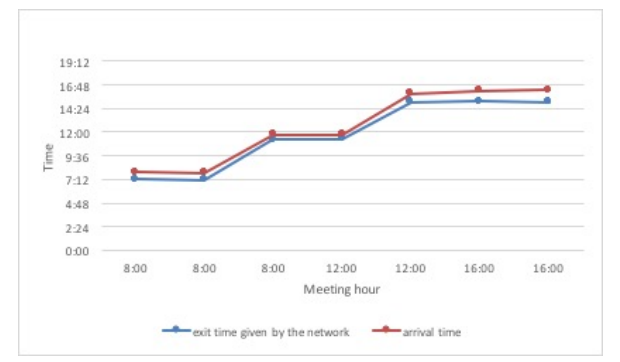

Fig. 14 Automatic alarm setup for SN3 neural network.

hours. It turns out that morning measurements are usually more accurate than those from a later hour. This may be due to the fact of traffic jams occurring in the city, which makes it harder to predict the return time.

Comparing SN1 results directly with SN2, we can conclude that in most cases, SN2 gave a slightly better result. In addition, some anomalies can be found, where the difference between the computed result and the real one is 6 minutes. Despite some shortcomings, both networks had a good tendency. Figures 12 - 13 show a comparison of actual time with that achieved through neural networks. Most of the time it was measured to within 3 minutes. This is a good result, and it can be safely said that in this case artificial intelligence methods such as neural networks are most useful in systems based on the IoT concept.

Table 2 and Fig. 14 present results for the automatic time setting service for the event. In this experiment, exactly the same route was selected, but it was reversed. Three different hours (meeting time) were examined as the target arrival time. After analyzing the results, we can conclude that with this problem the neural network has performed a bit worse than the first service, but it is still good enough to be able to consider these results as generally satisfactory. As we can see, at noon time of arrival was much shorter than that planned (even $15 \mathrm{~min}$ ahead of time). On the other hand, arriving at 16:00 in most cases resulted in delays. This fact may be caused, as above, by traffic jams in the city; neural networks have learned some inference that is not always immune to certain anomalies. This may mean that the network 
has too little training data and input parameters (increasing the dataset would improve the inference.)

\section{Conclusions}

In this paper we have investigated the complexity and usefulness of endowing IoT systems with artificial intelligence (AI). To that end, an IoT system, called smart-IoT, was built and several experiments were conducted to validate it with real data. The core AI methods are artificial neural networks with the reverse error propagation algorithm, although our approach can ne equally valid for using other AI methods, to be used in the context of reasoning. The built-in system uses mobile devices as smart objects and covers tow use cases, home-time return and automatic set-up of alarms for specific events. The study was conducted on a $4 \mathrm{~km}$ route and involved a comparison of the actual time achieved with those calculated by neural networks. In conclusion, although simple, our smart-IoT system is a full-fledged system based on artificial neural networks. It uses multiple micro-services for the artificial intelligence methods. For this reason, it is possible to expand it simply by other multiple AI methods in the context of a single service. This approach could increase the effect of inference with correct performance. Thus, the system is built in a modular way, so that upgrading to additional services can be seen as a core to IoT systems using artificial intelligence. Extending the system with more AI methods as well as addressing some security and privacy concerns $[5$ is our future work.

\section{References}

1. Anand, M., Susan, C., 2015. Artificial Intelligence Meets Internet of Things. Int. J. Sci. Eng. Comput. Technol. 5, 149.

2. Arsenio, A., Serra, H., Francisco, R., Nabais, F., Andrade, J., Serrano, E., 2014. Internet of intelligent things: Bringing artificial intelligence into things and communication networks, in: Xhafa, Bessis (Eds), Inter-Cooperative Collective Intelligence: Techniques and Applications. Springer, pp. 137.

3. Bari, N., Mani, G., Berkovich, S., 2013. Internet of things as a methodological concept, in: 4th Int'l Conf. on Computing for Geospatial Research and Application. IEEE, pp. 48-55.

4. Bessis, N., Xhafa, F., Varvarigou, D., Hill, R., Li, M., 2013. Internet of things and intercooperative computational technologies for collective intelligence. Springer.

5. Chasaki, D., Mansour, Ch, 2015. Security challenges in the internet of things. International Journal of Space-Based and Situated Computing, Vol. 5 No. 3, 141-149 doi:10.1504/IJSSC.2015.070945

6. Chen, F., Deng, P., Wan, J., Zhang, D., Vasilakos, A.V., Rong, X., 2015. Data mining for the internet of things: literature review and challenges. Int. J. Distrib. Sens. Netw. doi: doi.org/10.1155/2015/431047

7. Chiuchisan, I., Geman, O., 2014. An approach of a decision support and home monitoring system for patients with neurological disorders using internet of things concepts. WSEAS Transations Syst. 13, 460-469.

8. Cui, X., 2016. The internet of things, in: Ethical Ripples of Creativity and Innovation. Springer, pp. 61-68.

9. Da Xu, L., He, W., Li, S., 2014. Internet of things in industries: A survey. IEEE Trans. Ind. Inform. 10, 2233-2243. 
10. daCosta, F., 2013. Rethinking the Internet of Things. Apress, Berkeley, CA. doi:10.1007/978-1-4302-5741-7

11. Fan, Y.J., Yin, Y.H., Da Xu, L., Zeng, Y., Wu, F., 2014. IoT-based smart rehabilitation system. IEEE Trans. Ind. Inform. 10, 1568-1577.

12. Fortino, G., Guerrieri, A., Russo, W., Savaglio, C., 2014. Integration of agent-based and cloud computing for the smart objects-oriented iot, in: Proceedings of the IEEE 18th Int'l Conf. On Computer Supported Cooperative Work in Design (CSCWD). IEEE, pp. 493-498.

13. Gubbi, J., Buyya, R., Marusic, S., Palaniswami, M., 2013. Internet of Things (IoT): A vision, architectural elements, and future directions. Future Gener. Comput. Syst. 29, 1645-1660.

14. Holler, J., Tsiatsis, V., Mulligan, C., Avesand, S., Karnouskos, S., Boyle, D., 2014. From Machine-to-machine to the Internet of Things: Introduction to a New Age of Intelligence. Academic Press.

15. Hopfield, J.J., 2007. Hopfield network. Scholarpedia 2, 1977. doi:10.4249/scholarpedia.1977

16. Kelly, S.D.T., Suryadevara, N.K., Mukhopadhyay, S.C., 2013. Towards the implementation of IoT for environmental condition monitoring in homes. IEEE Sens. J. 13, 3846-3853.

17. Khan, R., Khan, S.U., Zaheer, R., Khan, S., 2012. Future internet: the internet of things architecture, possible applications and key challenges, in: 10th Int'l Conf. On Frontiers of Information Technology. IEEE, pp. 257-260.

18. Lin, N., Shi, W., 2014. The research on Internet of things application architecture based on web, in: 2014 IEEE Workshop on Advanced Research and Technology in Industry Applications (WARTIA), pp. 184-187. doi:10.1109/WARTIA.2014.6976227

19. Madakam, S., Ramaswamy, R., Tripathi, S., 2015. Internet of Things (IoT): A literature review. J. Comput. Commun. 3, 164.

20. Migliardi, M., Gaudina, M., Brogni, A., 2012. Pervasive services and mobile devices may support human memory and enhance daily efficiency. International Journal of SpaceBased and Situated Computing. Vol. 2 No. 3, 175-186. doi:10.1504/IJSSC.2012.048898

21. Miori, V., Russo, D., 2012. Anticipating health hazards through an ontology-based, IoT domotic environment, in: 6th Int'l Conf. On Innovative Mobile and Internet Services in Ubiquitous Computing. IEEE, pp. 745-750.

22. Morreale, P., Goncalves, A., Silva, C., 2015.Mobile ad hoc network communication for disaster recovery. International Journal of Space-Based and Situated Computing. Vol. 5 No. 3 178-186. doi:10.1504/IJSSC.2015.070949

23. OLeary, D.E., 2013. Artificial intelligence and big data. IEEE Intell. Syst. 28, 96-99.

24. Poniszewska-Maranda, A., Kaczmarek, D., 2015. Selected methods of artificial intelligence for Internet of Things conception, in: Federated Conf. On Computer Science and Information Systems. IEEE, pp. 1343-1348.

25. Settles, B., 2012. Active Learning. Synth. Lect. Artif. Intell. Mach. Learn. 6, 1-114. doi:10.2200/S00429ED1V01Y201207AIM018

26. Skouby, K.E., Lynggaard, P., 2014. Smart home and smart city solutions enabled by 5G, IoT, AAI and CoT services, in: Int'l Conf. On Contemporary Computing and Informatics. IEEE, pp. 874-878.

27. Sula, A., Spaho, E., Matsuo, K., Barolli, L., Xhafa, F., Miho, R., 2014 A new system for supporting children with autism spectrum disorder based on IoT and P2P technology. International Journal of Space-Based and Situated Computing. Vol. 4 No. 1, 55-64 doi:10.1504/IJSSC.2014.060688

28. Tan, L., Wang, N., 2010. Future internet: The internet of things, in: 3rd Int'l Conf. On Advanced Computer Theory and Engineering. IEEE, pp. V5-376.

29. Uckelmann, D., Harrison, M., Michahelles, F., 2011. Architecting the Internet of Things, 1st ed. Springer.

30. Valera, A.J.J., Zamora, M.A., Skarmeta, A.F., 2010. An architecture based on internet of things to support mobility and security in medical environments, in: 7th IEEE Consumer Communications and Networking Conf., pp. 1-5.

31. Vermesan, O., Friess, P., 2013. Internet of Things: Converging Technologies for Smart Environments and Integrated Ecosystems. River Publishers.

32. White, R.W., 1989. The artificial intelligence of urban dynamics: Neural network modelling of urban structure. Pap. Reg. Sci. 67, 43-53. 
33. Wu, M., Lu, T.-J., Ling, F.-Y., Sun, J., Du, H.-Y., 2010. Research on the architecture of Internet of Things, in: 3rd Int'l Conf. on Advanced Computer Theory and Engineering, pp. Vol. 5, 484-487. doi:10.1109/ICACTE.2010.5579493

34. Yun, M., Yuxin, B., 2010. Research on the architecture and key technology of Internet of Things (IoT) applied on smart grid, in: Int'l Conf. On Advances in Energy Engineering. IEEE, pp. 69-72.

35. Zhang, X.M., Zhang, N., 2011. An open, secure and flexible platform based on internet of things and cloud computing for ambient aiding living and telemedicine, in: Int'l Conf. On Computer and Management. IEEE, pp. 1-4. 